\title{
Numerical simulation of solitary waves on plane slopes
}

\author{
Philippe Guyenne ${ }^{\mathrm{a}, *}$, David P. Nicholls ${ }^{\mathrm{b}}$ \\ ${ }^{a}$ Department of Mathematics, McMaster University, 1280 Main Street West, Hamilton, Ont., Canada L8S 4 K1 \\ ${ }^{\mathrm{b}}$ Department of Mathematics, University of Notre Dame, Notre Dame, IN 46556, USA
}

Available online 2 March 2005

\begin{abstract}
In this paper, we present a numerical method for the computation of surface water waves over bottom topography. It is based on a series expansion representation of the Dirichlet-Neumann operator in terms of the surface and bottom variations. This method is computationally very efficient using the fast Fourier transform. As an application, we perform computations of solitary waves propagating over plane slopes and compare the results with those obtained from a boundary element method. A good agreement is found between the two methods.
\end{abstract}

(C) 2004 IMACS. Published by Elsevier B.V. All rights reserved.

Keywords: Solitary water waves; Dirichlet-Neumann operators; Bottom topography; Geometrical perturbation methods; Boundary element method

\section{Introduction}

Surface water wave propagation over variable depth has been studied for many years in applications to coastal engineering. This problem can be very complex due to the richness of coastal wave dynamics: when entering shallow water, waves are strongly affected by the bottom through shoaling, refraction, diffraction and reflection. Nonlinear effects related to wave-wave and wave-bottom interactions can cause wave scattering and depth-induced wave breaking. In turn, nonlinear waves can have a great influence on sediment transport and the formation of sandbars in nearshore regions.

\footnotetext{
* Corresponding author. Tel.: +1 905525 9140x27364; fax: +1 9055220935.

E-mail addresses: guyenne@math.mcmaster.ca (P. Guyenne); nicholls.2@nd.edu (D.P. Nicholls).
} 
Traditionally, the modeling of water waves over variable depth has been tackled using both the full Euler equations and long wave models such as shallow water equations or Boussinesq equations. Much work has been devoted to develop numerical methods for the solution to the full equations. Two main classes of numerical methods are boundary element methods $[18,10,15]$ and spectral methods. In particular, spectral methods based on perturbative expansions have been developed by Dommermuth and Yue [11], West et al. [25], Craig and Sulem [9], Nicholls [19] and Bateman et al. [2] for the modeling of water waves over constant or infinite depth. Similar methods have been proposed by Liu and Yue [17] and Smith [23] to include the effects of a bottom topography.

In this paper, we present a numerical method for the simulation of water waves over variable depth, based on an extension of the work of Craig and Sulem [9]. We use a series expansion formulation of the Dirichlet-Neumann operator on the free surface in terms of the surface and bottom variations. The spatial discretization is efficiently performed by a pseudospectral method using the fast Fourier transform. To test the accuracy of our method, we perform computations of solitary waves propagating over plane slopes and compare the results with those obtained from a boundary element method. For convenience, we restrict ourselves to a two-dimensional configuration.

The paper is organized as follows. In Section 2, we present the mathematical formulation of the problem and introduce the Dirichlet-Neumann operator. In Section 3, we give a description of the numerical method for the discretization of the equations. Section 4 describes the boundary element method, and in Section 5 , we present our numerical results of solitary waves propagating over a sloping bottom.

\section{Equations of motion and Dirichlet-Neumann operator}

We consider the motion of a free surface on top of a two-dimensional fluid domain defined by $\Omega(\beta, \eta)=$ $\{(x, y): x \in \mathbb{R},-h+\beta<y<\eta\}$, where $\beta(x)$ denotes the bottom perturbation and $\eta(x, t)$ denotes the free surface. The mean water level is located at $y=0$ and the constant reference depth is represented by $h$. The fluid is assumed to be incompressible, inviscid and irrotational, so that the fluid velocity is given by $u=\nabla \varphi$ and the velocity potential $\varphi(x, y, t)$ satisfies

$$
\Delta \varphi=0 \text { in } \Omega(\beta, \eta) .
$$

On the bottom boundary $y=-h+\beta$, the velocity potential obeys the Neumann boundary condition

$$
\partial_{n} \varphi=0,
$$

where $n$ is the exterior unit normal. The top boundary conditions are the usual kinematic and dynamic conditions imposed on $y=\eta$, namely

$$
\begin{aligned}
& \partial_{t} \eta=\partial_{y} \varphi-\left(\partial_{x} \eta\right)\left(\partial_{x} \varphi\right), \\
& \partial_{t} \varphi=-\frac{1}{2}|\nabla \varphi|^{2}-g \eta,
\end{aligned}
$$

where $g$ is the acceleration due to gravity. The effects of surface tension are neglected. Following [9], we set $\xi(x, t)=\varphi(x, \eta(x, t), t)$ and define the Dirichlet-Neumann operator

$$
G(\beta, \eta) \xi=\left.\left(1+\left(\partial_{x} \eta\right)^{2}\right)^{1 / 2} \partial_{n} \varphi\right|_{y=\eta},
$$

which maps Dirichlet data to Neumann data on the free surface. This operator is linear in $\xi$ but it is nonlinear with explicit nonlocal dependence on $\beta$ and $\eta$ which determine the fluid domain. In terms of 
the surface quantities $\eta$ and $\xi$, the boundary conditions (3) and (4) can be rewritten as

$$
\begin{aligned}
& \partial_{t} \eta=G(\beta, \eta) \xi \\
& \partial_{t} \xi=-\frac{1}{2\left(1+\left(\partial_{x} \eta\right)^{2}\right)}\left(\left(\partial_{x} \xi\right)^{2}-(G(\beta, \eta) \xi)^{2}-2\left(\partial_{x} \eta\right)\left(\partial_{x} \xi\right) G(\beta, \eta) \xi\right)-g \eta .
\end{aligned}
$$

These equations are Hamilton's canonical equations in Zakharov's formulation of the water wave problem as a Hamiltonian system [26], i.e.

$$
\partial_{t} \eta=\delta_{\xi} H, \quad \partial_{t} \xi=-\delta_{\eta} H
$$

with Hamiltonian

$$
H=\frac{1}{2} \int\left(\xi G(\beta, \eta) \xi+g \eta^{2}\right) \mathrm{d} x .
$$

The analyticity of Dirichlet-Neumann operators has been studied by many authors beginning with the work of Coifman and Meyer [4] which built on the results of Calderón [3]. Coifman and Meyer showed that, if $\eta \in \operatorname{Lip}(\mathbb{R})$, the Taylor series

$$
G(\beta, \varepsilon \eta)=\sum_{l=0}^{\infty} \varepsilon^{l} G^{(l)}(\beta, \eta)
$$

converges for $\varepsilon$ sufficiently small. Subsequently, for $\beta=0$, Craig et al. [8] extended these results for periodic $\eta \in C^{1}\left(\mathbb{R}^{2}\right)$, and Craig and Nicholls [6] generalized this argument for $\eta \in C^{1}\left(\mathbb{R}^{d}\right)$. Nicholls and Reitich [20] devised a direct method to estimate the $G^{(l)}$ (requiring the slightly stronger hypothesis $\eta \in$ $C^{3 / 2+\delta}\left(\mathbb{R}^{d}\right)$ ) with the goal of stabilized high-order calculations. These high-order results were exhibited in $[21,22]$, and joint analyticity results were established.

In the case of a flat bottom $(\beta=0)$, Craig and Sulem [9] showed that explicit expressions for the $G^{(l)}$ can be computed using a recursion formula, the operator expansion method. As noted by Craig and Nicholls [7], the adjoint recursion formula turns out to be more efficient in terms of computational time and memory storage, since each term $G^{(l)} \xi$ can be computed as a vector quantity. The first terms are given by

$$
\begin{aligned}
& G^{(0)}=D \tanh (h D), \quad G^{(1)}=D \eta D-G^{(0)} \eta G^{(0)}, \\
& G^{(2)}=\frac{1}{2}\left(G^{(0)} D \eta^{2} D-D^{2} \eta^{2} G^{(0)}-2 G^{(0)} \eta G^{(1)}\right),
\end{aligned}
$$

where $D=-\mathrm{i} \partial_{x}$ and $G^{(0)}$ represent Fourier multiplier operators.

This approach can be extended to account for a nontrivial bottom topography. In Craig et al. [5], it is shown that the same recursion formula as in (11) can be written for the Dirichlet-Neumann operator, except that the first term $G^{(0)}$ is replaced by

$$
G^{(0)}=D \tanh (h D)+D L(\beta) .
$$

By an extension of the argument in [20], it can be shown that the operator $L(\beta)$ can be expressed as a convergent Taylor series

$$
L(\beta)=\sum_{j=0}^{\infty} L_{j}(\beta),
$$


whose terms can be also derived explicitly from a recursion formula. The first terms are given by

$$
L_{0}=0, \quad L_{1}=-\operatorname{sech}(h D) \beta \operatorname{sech}(h D), \quad L_{2}=\operatorname{sech}(h D) \beta D \sinh (h D) L_{1},
$$

where the $L_{j}$ are homogeneous of degree $j$ in $\beta$. Clearly, $L(\beta)=0$ for $\beta=0$ and $G(0, \eta)$ reduces to that for a flat bottom. The series expansion representation of the Dirichlet-Neumann operator as presented here is suitable for numerical computations (see Section 3) and it proves useful for the systematic derivation of Hamiltonian long wave approximations with bottom topography. The reader is referred to [5] for more details on the derivation of $L(\beta)$ and on the long wave expansions.

\section{Numerical method}

We assume periodic boundary conditions in the $x$-direction and, as in $[9,19]$, we use a pseudospectral method for the space discretization of the problem. This is a natural choice for the computations of $G(\beta, \eta)$ and $L(\beta)$ since each term in (11) and (14) consists of concatenations of Fourier multipliers with powers of $\beta$ and $\eta$. Both operators are approximated by a finite number of terms

$$
G(\beta, \eta) \simeq \sum_{l=0}^{M_{G}} G^{(l)}(\beta, \eta), \quad L(\beta) \simeq \sum_{j=0}^{M_{L}} L_{j}(\beta),
$$

where the orders $M_{G}$ and $M_{L}$ are independently chosen according to the physical problem under consideration. In practice, it is not necessary to specify large values of $M_{G}$ and $M_{L}$ due to the fast convergence of the series expansions for $G(\beta, \eta)$ and $L(\beta)$. The three variables $\eta, \xi$ and $\beta$ are expanded in truncated Fourier series with the same number of modes. Applications of Fourier multipliers are performed in spectral space, while nonlinear products are calculated in physical space at a discrete set of equally spaced points. All operations are performed via the FFTW routines [12].

Time integration is carried out in Fourier space. The linear terms in (6) and (7) are exactly solved by an integrating factor technique. The nonlinear terms are integrated using a fourth-order AdamsBashford/Moulton predictor-corrector scheme with constant time step [2]. A predictor-corrector scheme is preferred for example over a Runge-Kutta scheme [11,17] because the latter would require multiple evaluations of $G(\beta, \eta)$ at intermediate times, which results in a larger computational effort and a faster accumulation of numerical errors. On the other hand, the predictor-corrector scheme requires more memory storage since one needs to save the solution at some previous times. This is however not too memory demanding at least for two-dimensional problems.

In our computations, after some time of integration, we have observed spurious oscillations in the wave profile due to an onset of instability initiated by some growth of numerical errors at high wavenumbers. The cause of this problem may be related to aliasing errors inherent to the pseudospectral method and/or the ill-conditioning of the operator expansion method [21]. Similar high-wavenumber instabilities were observed by other authors (e.g. $[9,11,18]$ ) who used smoothing techniques to overcome this difficulty. Here, at every time step, we apply an ideal low-pass filter to $\eta$ and $\xi$ of the form

$$
f(k)= \begin{cases}1 & \text { if } \frac{|k|}{k_{\max }} \leq v, 0<v \leq 1, \\ 0 & \text { if } \frac{|k|}{k_{\max }}>v,\end{cases}
$$


where $k_{\max }$ is the highest wavenumber of the spectrum. In our applications, we typically found that $v=0.8$ suffices to stabilize the solution. Applying an ideal filter with a high value of $v$ ensures that only very low energy levels located in the high-wavenumber region of the spectrum are suppressed by filtering.

\section{Boundary integral formulation and boundary element method}

In this section, we briefly describe the numerical model developed by Grilli et al. [15] for the simulation of two-dimensional nonlinear water waves over bottom topography. This model has been recently extended to three dimensions by Grilli et al. [13].

The governing equations are still given for a potential flow. However, the kinematic and dynamic boundary conditions (3) and (4) are expressed in the mixed Eulerian-Lagrangian formulation as

$$
\begin{aligned}
& \mathrm{d}_{t} x=u=\nabla \varphi, \\
& \mathrm{d}_{t} \varphi=\frac{1}{2}|\nabla \varphi|^{2}-g y,
\end{aligned}
$$

where $\mathrm{d} / \mathrm{d} t=\partial / \partial t+\nabla \varphi \cdot \nabla$ denotes the Lagrangian time derivative and $x$ denotes the vector position of a fluid particle on the free surface. Green's second identity transforms Laplace's equation (1) into the boundary integral equation

$$
\alpha(x) \varphi(x)=\int_{S}\left(\partial_{n} \varphi\left(x^{\prime}\right) \Gamma\left(x^{\prime}, x\right)-\varphi\left(x^{\prime}\right) \partial_{n} \Gamma\left(x^{\prime}, x\right)\right) \mathrm{d} S^{\prime},
$$

where $\alpha(x)$ is proportional to the exterior solid angle at point $x$ and $\Gamma\left(x^{\prime}, x\right)$ is the two-dimensional free space Green's function defined by

$$
\Gamma\left(x^{\prime}, x\right)=-\frac{1}{2 \pi} \ln \left|x^{\prime}-x\right|,
$$

which depends on the distance from the source point $x^{\prime}$ to the field point $x$, both on the boundary $S$. Note that, in three dimensions, the Green's function is defined by $\Gamma\left(x^{\prime}, x\right)=1 /\left(4 \pi\left|x^{\prime}-x\right|\right)$. The boundary $S$ of the fluid domain includes the free surface, the bottom and the lateral boundaries. Eq. (19) corresponds to the boundary integral formulation of the Dirichlet-Neumann operator, as opposed to the perturbative formulation given in Section 2.

Grilli et al. [15] used a high-order boundary element (BE) method for the discretization of (19). The boundary is discretized into collocation nodes, defining elements for local interpolation of the solution in between these nodes. The boundary elements are four-node quadrilaterals associated with cubic shape functions. The discretized boundary integrals are evaluated by Gauss-Legendre quadrature. A method of singularity extraction based on coordinate transformations is used to calculate the integrals at singular points. The linear algebraic system resulting from the discretization of (19) is numerically solved either by a direct method (LU decomposition) or, for large-scale problems, by an iterative method (GMRES). The evolution equations (17) and (18) are integrated in time using a second-order explicit scheme based on Taylor series expansions of the variables $x$ and $\varphi$ about the current time. The advantages of this scheme are that multiple solutions to the linear system are not required at intermediate times and, once the linear system is solved, the matrix associated with it can be used to compute the high-order terms in the temporal Taylor expansions without too much additional effort (see also [10]). 
It should be noted that this two-dimensional BE model has been extensively tested and validated against experimental results for both solitary waves and periodic waves [14-16]. The reader is referred to [13,15] for more details on their numerical models. In this paper, we use the BE model as a reference to test the performance of our operator expansion (OE) model.

\section{Numerical results}

The numerical method described in Section 3 is used to compute the evolution of solitary waves traveling up plane slopes. The accuracy of the computations are assessed by direct comparison with results obtained from the BE method described in Section 4.

The geometry of the bottom is specified as follows: a flat bottom of depth $h$ over a short distance $d$ from the left extremity of the domain, and a slope $s$ for $x \geq d$. All physical quantities are non-dimensionalized according to long wave theory, i.e. lengths are divided by $h$ and times divided by $\sqrt{h / g}$. This is equivalent to setting $g=1$ and $h=1$. The initial condition is a fully nonlinear solitary wave of height $A$ computed by Tanaka's method [24]. This corresponds to a numerically exact and permanent solution of potential flow theory. We have checked numerically that such a wave propagates with negligible change of shape and speed on uniform depth in our model. Because fixed vertical walls are specified at extremities of the domain in the BE model, both the bottom topography and the initial condition in the OE code are symmetrically extended by reflection in order to simulate a Neumann boundary condition at some distance up the slope. As shown in Fig. 1, the situation is two solitary waves propagating symmetrically towards a submerged island between them. For the computations presented below, we have used expansions of $G(\beta, \eta)$ and $L(\eta)$ up to orders $M_{G}=8$ and $M_{L}=2$ which give satisfactory results with reasonable cost.

We present results of computations for incident solitary waves of amplitudes $A=0.2,0.3,0.4$. In the three cases, the imposed bottom slope is fairly steep $(s=1 / 18)$. It starts at $x=d=8.225$ and ends at $x=25$. A resolution of 512 points over the whole domain $0 \leq x \leq 52$ was specified in the pseudospectral code. The time step was chosen sufficiently small: $\Delta t=0.01$ for $A=0.2,0.3$ and $\Delta t=0.005$ for $A=0.4$. Fig. 1 actually shows the configuration with $A=0.2$ and $s=1 / 18$. In the BE model, the domain is truncated at $x=25$ and a discretization of 128 nodes was specified for the free surface (and

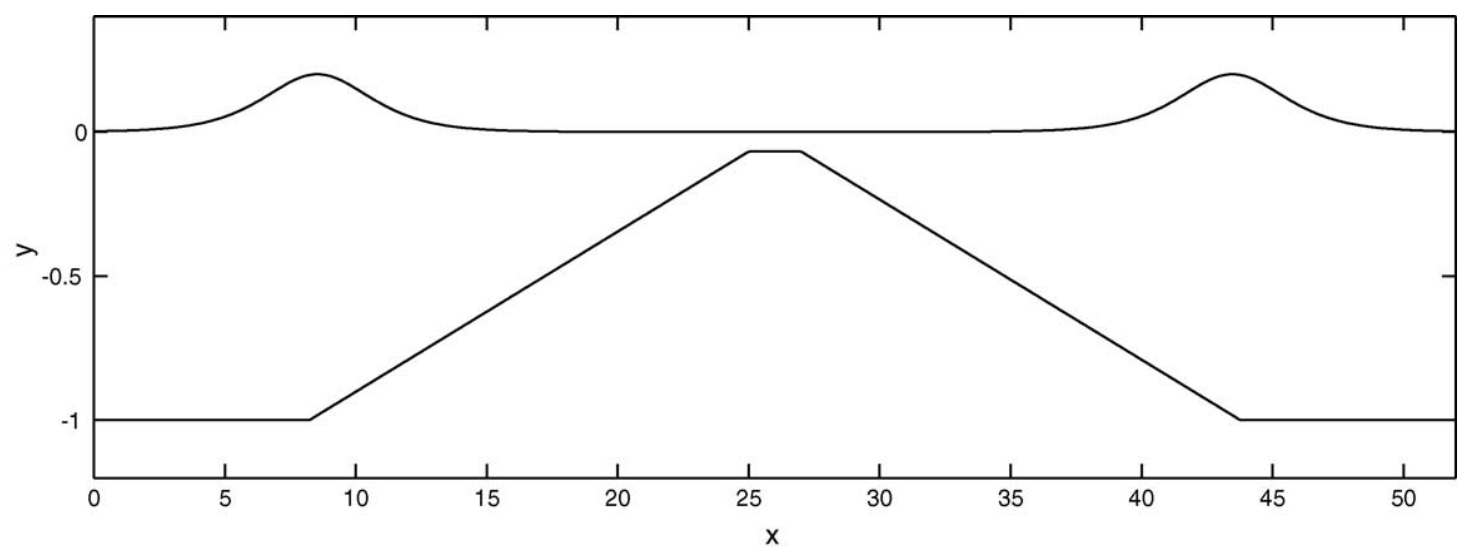

Fig. 1. Sketch of the bottom topography and initial condition in the OE model. Here the two solitary waves are of amplitude $A=0.2$ and the bottom slope is $s=1 / 18$. 

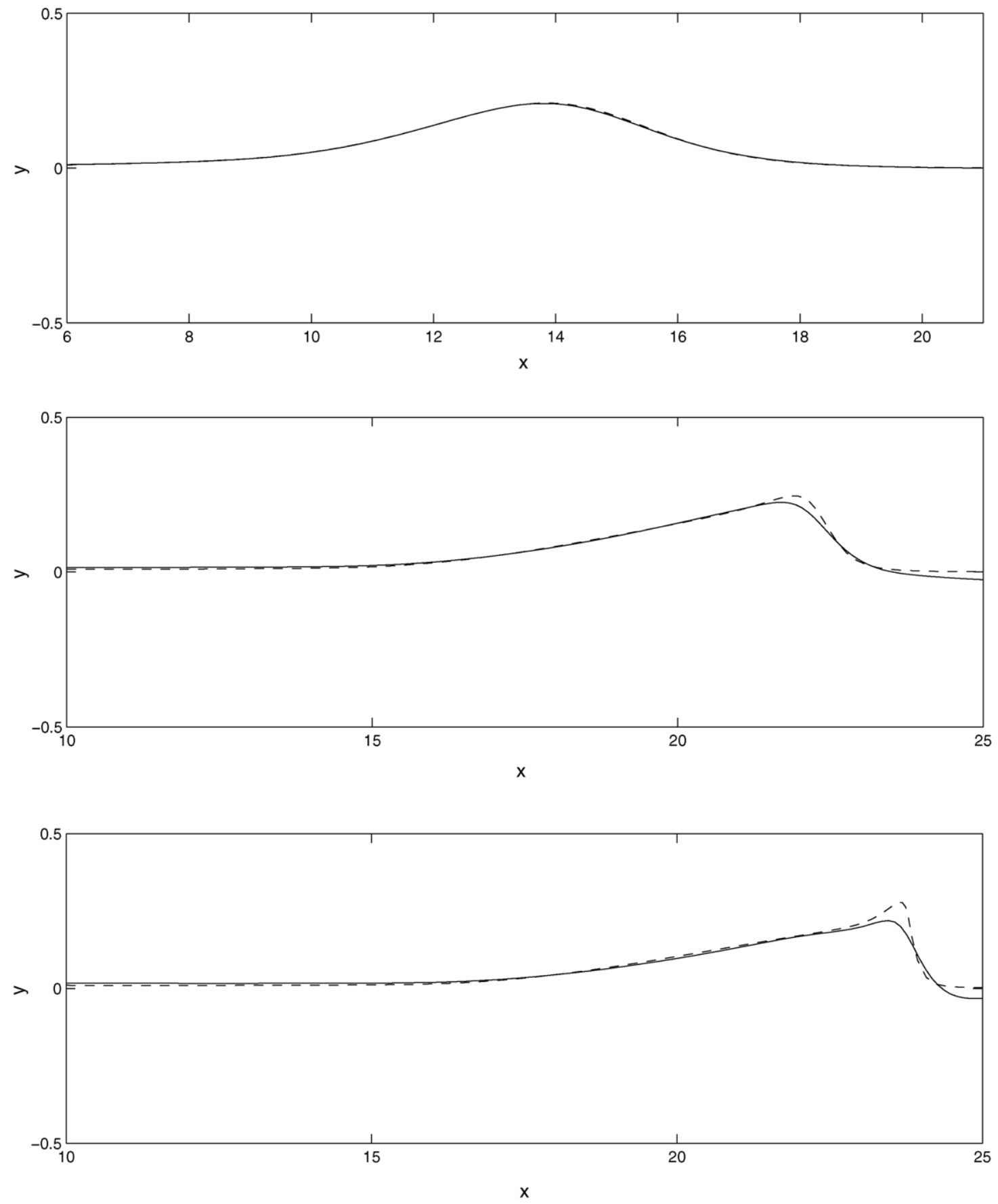

Fig. 2. Comparison between BE (dashed line) and OE (solid line) wave profiles for $s=1 / 18$ and $A=0.2$ at $t=5,13,14.8$ (from top to bottom). 

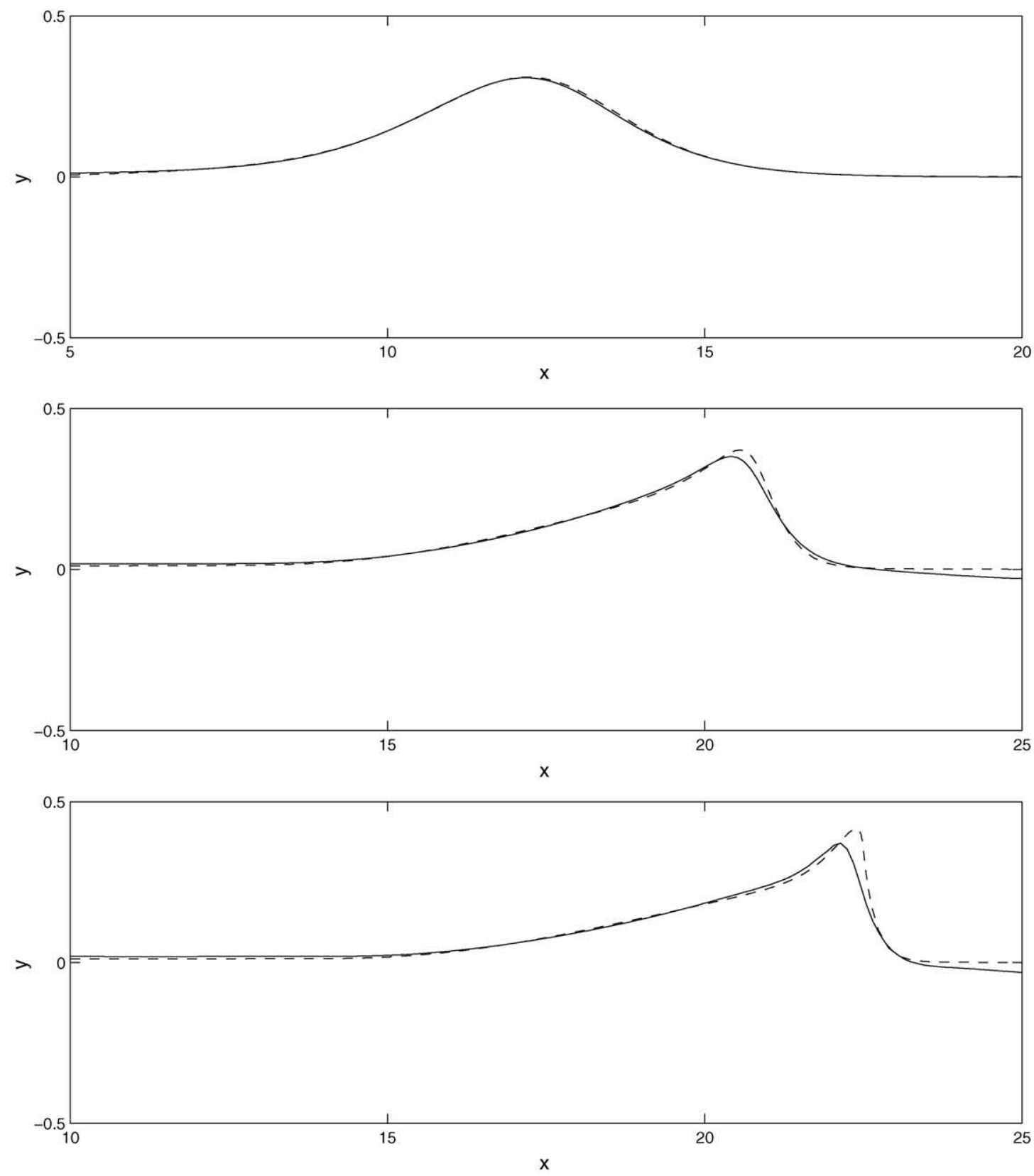

Fig. 3. Comparison between BE (dashed line) and OE (solid line) wave profiles for $s=1 / 18$ and $A=0.3$ at $t=4.4,12,13.6$ (from top to bottom).

the bottom). Note that it is not necessary to use a very large number of nodes because, in the mixed Eulerian-Lagrangian formulation, nodes which are associated with fluid particles move according to the fluid velocity and tend to gather in regions of large gradients. As a result, there is a gain of resolution in small-scale regions like the wave crest which need to be well-resolved. 

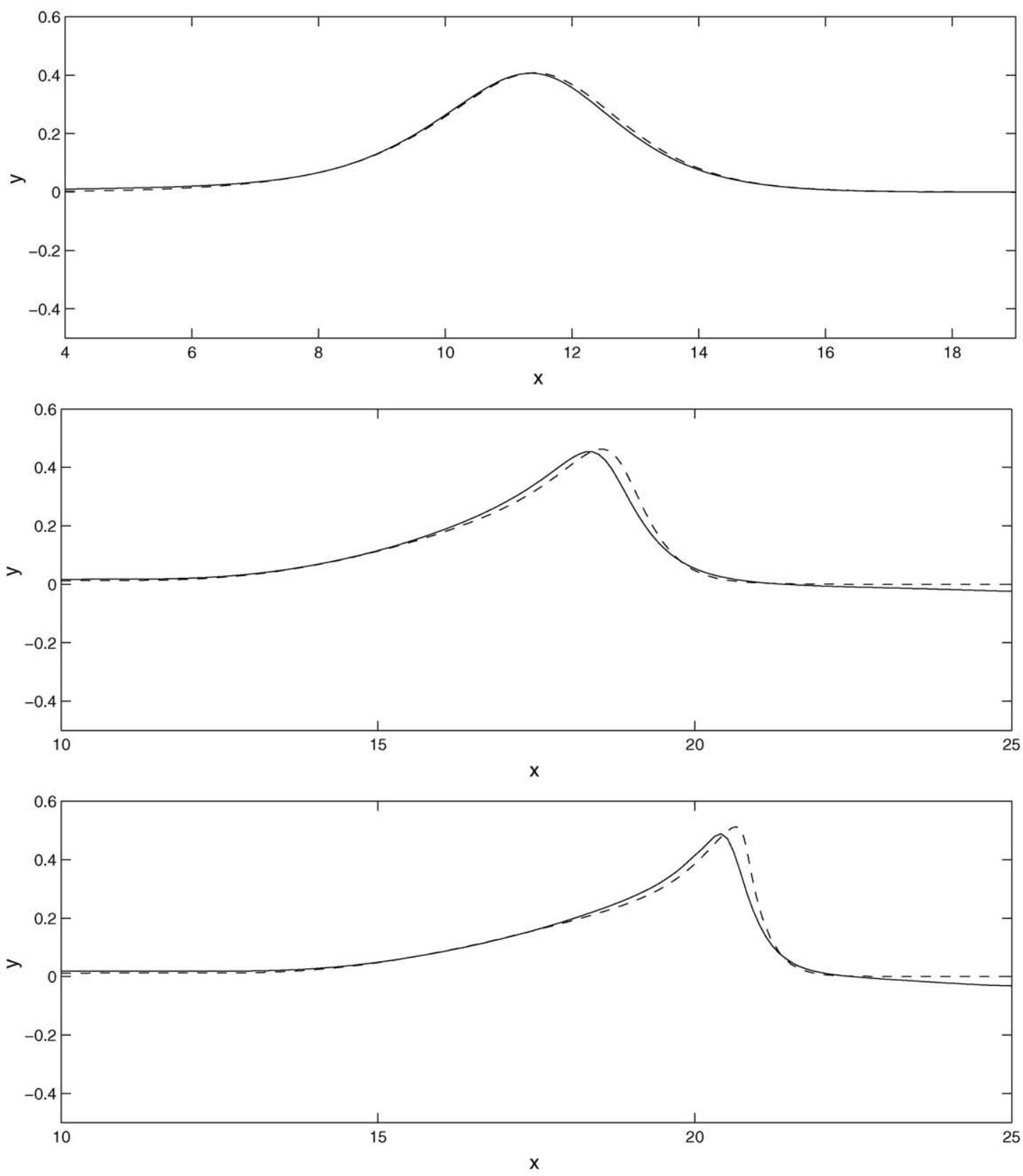

Fig. 4. Comparison between BE (dashed line) and OE (solid line) wave profiles for $s=1 / 18$ and $A=0.4$ at $t=4.2,10.2,12$ (from top to bottom).

Figs. 2-4 show the comparison of wave profiles at three successive times for $A=0.2,0.3,0.4$ respectively. For the OE computations, only the left half of the domain is displayed. One can see that, at early times, the BE and OE solutions remain indistinguishable. However, as the solitary waves travel up the slope and steepen, some discrepancies become noticeable, especially at and ahead of the wave 


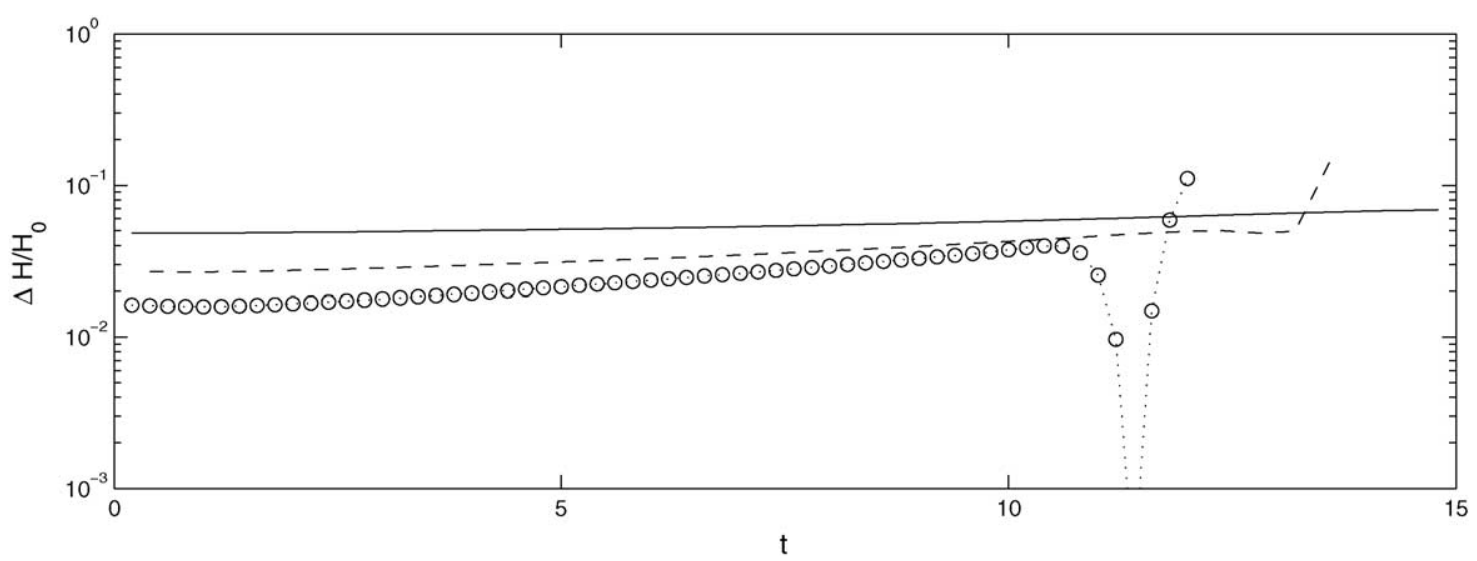

Fig. 5. Time evolution of relative errors of energy conservation $\Delta H / H_{0}$ for $A=0.2$ (solid line), 0.3 (dashed line) and 0.4 (circles) in the $\mathrm{OE}$ model with $s=1 / 18 . H_{0}$ denotes the energy level at the initial time.

crest. The BE solution exhibits a sharper and higher wave crest than that of the OE solution. A slightly higher surface elevation is also noticeable ahead of the wave for the BE solution. These discrepancies are more pronounced in the latest stages as the solitary waves get close to the breaking point after having propagated some distance up the slope. The breaking point corresponds to the instant when the front face of the wave reaches a vertical tangent before the wave crest overturns. Increasing the resolution in the spectral computations does not yield a better agreement between BE and OE results. It should be remembered that the Eulerian-Lagrangian BE formulation allows for wave overturning while the $\mathrm{OE}$ method requires that the free surface be represented as the graph of a function, $y=\eta(x, t)$. Consequently, it is not surprising that near the onset of breaking the BE results are more reliable. Nevertheless, the OE results are quite close to the $\mathrm{BE}$ ones until near the time of breaking. One can see that, for the three cases $A=0.2,0.3,0.4$, the rear faces of the waves match almost perfectly and the wave crests have nearly the same locations. As expected, the higher the incident wave amplitude is, the sooner the steepening process occurs.

Although there is a small difference of locations for the Neumann boundary condition at the right end of the slope between the two models, we have checked that reducing the length of the plateau on top of the submerged island in the OE code, or extending the truncation of the domain in the BE code, gives similar results as previously. Solitary waves have a velocity distribution which is very localized around the wave crest, and thus the Neumann boundary condition plays a small role until the boundary is reached.

In Fig. 5, we plot the relative errors of energy conservation

$$
\frac{\Delta H}{H_{0}}=\frac{H-H_{0}}{H_{0}},
$$

for $A=0.2,0.3,0.4$ in the OE model, where $H$ is given by (9) and $H_{0}$ denotes the energy level at the initial time. Overall, the errors remain small (less than 10\%) until the latest stages of wave shoaling shown in Figs. 2-4. As expected, there is a gradual growth of errors as the waves approach breaking. One can also see that the relative errors are smaller for high-amplitude waves than for small-amplitude waves. This is in accordance with the results in Figs. 2-4 which show a better agreement between BE and OE models for high wave amplitudes. A possible explanation is that high-amplitude waves are less influenced 

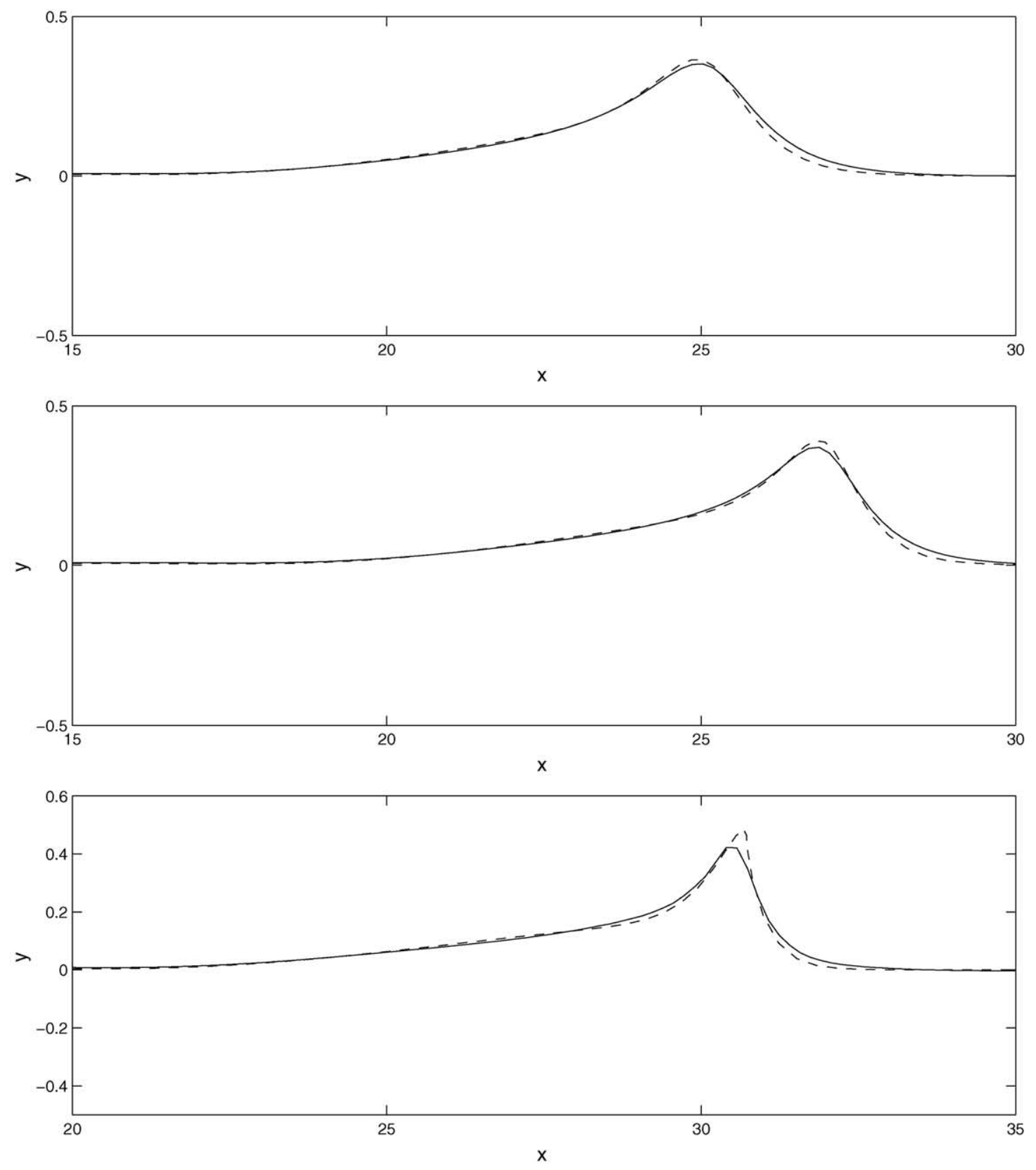

Fig. 6. Comparison between BE (dashed line) and OE (solid line) wave profiles for $s=1 / 35$ and $A=0.3$ at $t=16,17.8,21.4$ (from top to bottom).

by the bottom, especially at early stages of shoaling, and so they are less affected by errors due to the approximation of the bottom topography.

Finally, Fig. 6 shows the comparison between $\mathrm{BE}$ and $\mathrm{OE}$ results for $A=0.3$ and a milder bottom slope $s=1 / 35$. The latter extends over a longer interval $8.225 \leq x \leq 42$ but the same discretizations as 
previously are specified for the two models. Similar observations as for Fig. 3 can be made, except that the steepening process takes longer to develop. Nevertheless, it can be seen that the two solutions remain close to each other over a long time of integration.

\section{Conclusions}

We have presented a numerical method for the computation of unsteady nonlinear surface waves over bottom topography. As an extension of the work of Craig and Sulem [9], the model is based on the expansion of the Dirichlet-Neumann operator in terms of the surface and bottom variations and a pseudospectral method is used for the spatial discretization of the problem. In this paper, we have applied the model to the computation of solitary waves propagating over plane slopes and direct comparisons with results from a boundary element method have been made. Near breaking, the operator expansion representation is numerically not as robust as the boundary element method, since questions arise concerning the stability of operator expansion formulas and limitations of the underlying formulation in terms of the free surface as the graph of a function. Nevertheless, we have obtained quite satisfactory results of wave shoaling up to the time of breaking; a good agreement is found for the amplitude and location of the wave crest. Regarding the computational efficiency, it should be emphasized that the present formulation takes advantage of the fast Fourier transform and, unlike the boundary element method, no additional collocation points are required for the discretization of the bottom. Our method can also be easily extended to three dimensions as well as to moving (time-dependent) bottom geometries. For the near future, we plan to address the problems of wave transformations over submerged bars and sinusoidal ripples, with different types of lateral boundary conditions like wavemakers and absorbing beaches [1,23].

\section{Acknowledgements}

The authors thank W. Craig, C. Sulem and S.T. Grilli for helpful advice. P.G. acknowledges financial support and disposal of computing resources from SHARCNET. D.P.N. gratefully acknowledges support from NSF through grant nos. DMS-0196452 and DMS-0139822.

\section{References}

[1] Y. Agnon, H.B. Bingham, A non-periodic spectral method with application to nonlinear water waves, Eur. J. Mech. B Fluids 18 (1999) 527-534.

[2] W.J.D. Bateman, C. Swan, P.H. Taylor, On the efficient numerical simulation of directionally spread surface water waves, J. Comput. Phys. 174 (2001) 277-305.

[3] A.P. Calderón, Cauchy integrals on Lipschitz curves and related operators, Proc. Natl. Acad. Sci. USA 75 (1977) $1324-1327$.

[4] R. Coifman, Y. Meyer, Nonlinear harmonic analysis and analytic dependence, in: Proceedings of the Conference on Pseudodifferential Operators and Applications, Notre Dame, IN, 1984, Amer. Math. Soc., 1985, pp. 71-78.

[5] W. Craig, P. Guyenne, D.P. Nicholls, C. Sulem, Hamiltonian long wave expansions for water waves over a rough bottom, Proc. R. Soc. A 461 (2005) 839-873.

[6] W. Craig, D.P. Nicholls, Traveling two and three dimensional capillary gravity water waves, SIAM J. Math. Anal. 32 (2) (2000) 323-359. 
[7] W. Craig, D.P. Nicholls, Traveling gravity water waves in two and three dimensions, Eur. J. Mech. B Fluids 21 (2002) 615-641.

[8] W. Craig, U. Schanz, C. Sulem, The modulation regime of three-dimensional water waves and the Davey-Stewartson system, Ann. Inst. Henri Poincaré 14 (1997) 615-667.

[9] W. Craig, C. Sulem, Numerical simulation of gravity waves, J. Comput. Phys. 108 (1993) 73-83.

[10] J.W. Dold, D.H. Peregrine, An efficient boundary integral method for steep unsteady water waves, in: K.W. Morton, M.J. Baines (Eds.), Numer. Meth. for Fluid Dynamics II, 1986, pp. 671-679.

[11] D.G. Dommermuth, D.K.P. Yue, A high-order spectral method for the study of nonlinear gravity waves, J. Fluid Mech. 184 (1987) 267-288.

[12] M. Frigo, S.G. Johnson, The fastest Fourier transform in the West, MIT-LCS-TR-728. http://www.theory.lcs.mit.edu/fftw/.

[13] S.T. Grilli, P. Guyenne, F. Dias, A fully nonlinear model for three-dimensional overturning waves over arbitrary bottom, Int. J. Numer. Meth. Fluids 35 (2001) 829-867.

[14] S.T. Grilli, J. Horrillo, Numerical generation and absorption of fully nonlinear periodic waves, J. Eng. Mech. 123 (1997) 1060-1069.

[15] S.T. Grilli, R. Subramanya, I.A. Svendsen, J. Veeramony, Shoaling of solitary waves on plane beaches, J. Waterway Port Coastal Ocean Eng. 120 (1994) 609-628.

[16] S.T. Grilli, I.A. Svendsen, R. Subramanya, Breaking criterion and characteristics for solitary waves on slopes, J. Waterway, Port, Coastal, Ocean Eng. 123 (1997) 102-112.

[17] Y. Liu, D.K.P. Yue, On generalized Bragg scattering of surface waves by bottom ripples, J. Fluid Mech. 356 (1998) 297-356.

[18] M.S. Longuet-Higgins, E.D. Cokelet, The deformation of steep surface waves on water. I. A numerical method of computation, Proc. Roy. Soc. Lond. A 350 (1976) 1-26.

[19] D.P. Nicholls, Traveling water waves: spectral continuation methods with parallel implementation, J. Comput. Phys. 143 (1998) 224-240.

[20] D.P. Nicholls, F. Reitich, A new approach to analyticity of Dirichlet-Neumann operators, Proc. Roy. Soc. Edin. Sect. A 131 (6) (2001) 1411-1433.

[21] D.P. Nicholls, F. Reitich, Stability of high-order perturbative methods for the computation of Dirichlet-Neumann operators, J. Comput. Phys. 170 (2001) 276-298.

[22] D.P. Nicholls, F. Reitich, Analytic continuation of Dirichlet-Neumann operators, Numer. Math. 94 (1) (2003) 107-146.

[23] R. Smith, An operator expansion formulation for nonlinear surface water waves over variable depth, J. Fluid Mech. 363 (1998) 333-347.

[24] M. Tanaka, The stability of solitary waves, Phys. Fluids 29 (1986) 650-655.

[25] B.J. West, K.A. Brueckner, R.S. Janda, D.M. Milder, R.L. Milton, A new numerical method for surface hydrodynamics, J. Geophys. Res. 92 (1987) 11803-11824.

[26] V.E. Zakharov, Stability of periodic waves of finite amplitude on the surface of a deep fluid, J. Appl. Mech. Tech. Phys. 9 (1968) 190-194. 